\title{
Noninvasive tool for the diagnosis of NAFLD in association with atherosclerotic cardiovascular risk
}

\author{
Nevine I. Musa ${ }^{1 *}$, Eslam Safwat ${ }^{1}$, Sara M. Abdelhakam² ${ }^{2}$ Amir M. Farid ${ }^{2}$ and Waleed M. Hetta ${ }^{3}$
}

\begin{abstract}
Background: Whether the severity of liver histology in non-alcoholic fatty liver disease (NAFLD) is associated with more pronounced cardiovascular disease is unsettled. There is a need to develop a noninvasive tool to help its diagnosis in association with atherosclerotic cardiovascular disease. We aimed to evaluate the diagnostic performance of NAFLD-liver fat score (NAFLD-LFS) and carotid intima-media thickness (CIMT) in magnetic resonance imagingproved NAFLD. The study comprised 60 patients with NAFLD during the period from October 2015 to June 2017, diagnosed by clinical features, laboratory tests, and magnetic resonance study. Thirty healthy subjects served as controls. All included individuals were subjected to anthropometric measurements and measurement of NAFLD-LFS and CIMT.

Results: On doing ultrasonography, 30 cases showed mild, 24 showed moderate, and 6 cases showed severe steatosis. NAFLD-LFS at a cutoff value of -1.628 showed a sensitivity of $96.7 \%$, specificity $100 \%$, positive predictive value $100 \%$, negative predictive value $93.8 \%$, and accuracy $97.8 \%$. CIMT at a cutoff value of 0.6 had a sensitivity of $70 \%$, specificity $53.3 \%$, positive predictive value $75 \%$, negative predictive value $47.1 \%$, and accuracy $64.4 \%$. The combination of CIMT at cutoff 0.7 and NAFLD-LFS at cutoff - 1.628 showed sensitivity, specificity, positive predictive value, negative predictive value, and diagnostic accuracy of $100 \%$.
\end{abstract}

Conclusion: CIMT combined with NAFLD-LFS can produce a simple noninvasive tool for diagnosis of NAFLD.

Keywords: Non-alcoholic fatty liver disease, Noninvasive, Liver fat score, Carotid intima-media thickness, Magnetic resonance imaging

\section{Background}

It is well-known nowadays that non-alcoholic fatty liver disease (NAFLD) is the most prevalent chronic liver disease worldwide. NAFLD spectrum includes hepatic steatosis, non-alcoholic steatohepatitis (NASH), and hepatic fibrosis up to cirrhosis [1]. Patients with NAFLD are usually incidentally discovered on doing routine liver enzymes or abdominal imaging [2]. Mortality is estimated to be about 10 to $20 \%$ after 10 to 15 years of diagnosis, while the risk of progression to cirrhosis is $5-10 \%$ and $1-2 \%$ to $\mathrm{HCC}$ [3].

NAFLD is a hepatic manifestation of the metabolic syndrome (MS), including type 2 diabetes mellitus (DM)

\footnotetext{
* Correspondence: nevine_musa@yahoo.com

'Department of Internal Medicine, Faculty of Medicine, Ain Shams University, Khalifa El-Maamon St., Abbassia, Cairo 11341, Egypt

Full list of author information is available at the end of the article
}

and cardiovascular disease (CVD) [4]. Recently, a systematic review declared that NAFLD patients are prone to develop CVD, even without MS. Moreover, atherosclerotic cardiovascular disease seems to play an important role in the natural course of NAFLD. Therefore, there is an urgent need to develop and validate a reproducible noninvasive diagnostic tool that can accurately grade the severity of liver disease and its progression in NAFLD patients in correlation with cardiovascular atherosclerotic disease [5]. The carotid intima-media thickness (CIMT) parallels the importance of other cardiovascular risk factors [6].

It is unreasonable to use liver biopsy routinely to diagnose NAFLD, attributed to its limitations including cost, invasiveness, complications, and inter-observer variability [7]. Thus, it seems more logic to use ultrasound as an available, convenient, safe, and relatively inexpensive imaging 
tool. However, its sensitivity is limited when steatosis is less than $30 \%$ on liver biopsy [8].

Magnetic resonance imaging (MRI) including spectroscopy has higher sensitivity and specificity in quantifying steatosis [9]. But these studies are time-consuming, relatively expensive, and often unavailable in daily routine. So, more simple tests have been developed based on routine laboratory and anthropometric parameters, including fatty liver index (FLI), the hepatic steatosis index (HSI), and the NAFLD-liver fat score (NAFLD-LFS) [10]. NAFLD-LFS includes AST/ALT ratio [11].

The present study was designed to evaluate the performance of NAFLD-liver fat score (NAFLD-LFS) and carotid intima-media thickness (CIMT) in predicting MRI-diagnosed NAFLD.

\section{Methods}

The current study included 60 adult patients older than 18 years with MRI-proved NAFLD, who were randomly recruited from the outpatient clinic of hepatology at Internal Medicine and tropical departments during the period from October 2015 to June 2017. Additionally, a total of 30 age- and sex-matched healthy subjects negative for any systemic or hepatic diseases were included in this study as controls.

Patients with history of ischemic heart disease, use of steatosis-inducing medications (e.g. amiodarone), selfreporting alcohol consumption of $>20 \mathrm{~g} /$ day for women and $>30 \mathrm{~g} /$ day for men, and metabolic causes of steatohepatitis including Wilson's disease, hemochromatosis, viral hepatitis $\mathrm{B}$ or $\mathrm{C}$, and autoimmune liver diseases were excluded from the study.

The study was conducted in accordance with the ethical principles of the 1975 Declaration of Helsinki and was approved by Research Ethics Committee. A written informed consent was obtained from all the participants after explaining the aim and concerns of the study.

All the included individuals were subjected to a thorough medical history and clinical examination with special emphasis on blood pressure measurement and anthropometric measures including patient's weight to the nearest $0.1 \mathrm{~kg}$ and height to the nearest $0.1 \mathrm{~cm}$. The body mass index (BMI) was calculated as follows: weight (in kilograms) divided by square of height (in meters); BMI of $30 \mathrm{~kg} / \mathrm{m}^{2}$ or more was defined as obesity.

All anthropometric measures were applied in the morning. The waist circumference was measured midway between the lowest rib and the top of the iliac crest at the end of normal expiration. The hip circumference was measured in a horizontal plane at the maximum extension of the buttocks. The waist/hip ratio $(\mathrm{W} / \mathrm{H})$ was calculated as the waist measurement divided by the hip measurement. Resting electrocardiographic (ECG) and echocardiographic assessment was conducted.
Routine laboratory investigations were done including complete blood count $(\mathrm{CBC})$, liver, renal, and lipid profiles. Also, HBs Ag, HCV Ab, serum iron, ferritin, ceruloplasmin, anti-nuclear antibody (ANA), and anti-smooth muscle antibody (ASMA) were done. Fasting blood glucose (FBG) and fasting insulin (FI) were tested after $8 \mathrm{~h}$ fasting. FBG $>126 \mathrm{mg} / \mathrm{dl}$ or $\mathrm{HbA} 1 \mathrm{c} \geq 6.5 \%$ was defined as DM [12].

Insulin resistance (IR) was calculated by using the homeostasis model assessment-insulin resistance equation: [HOMA-IR = plasma glucose $(\mathrm{mg} / \mathrm{dl}) \times$ insulin $(\mathrm{uU} /$ $\mathrm{ml}) / 405]$, a value of $>2.5$ is the cutoff of IR [13]. MS was defined according to the Joint Scientific Statement 2009 [14], as any three of the following: waist circumference $\geq 102 \mathrm{~cm}$ in males or $\geq 88 \mathrm{~cm}$ in females, triglycerides $\geq 150 \mathrm{mg} / \mathrm{dl}$, HDL $<40 \mathrm{mg} / \mathrm{dl}$ in males or $<50 \mathrm{mg} / \mathrm{dl}$ in females, FBG $\geq 100 \mathrm{mg} / \mathrm{dl}$, or blood pressure $\geq 130 / 85 \mathrm{mmHg}$. Measurement of NAFLD-LFS was calculated as follows: $-2.89+1.18 \times$ MS $($ Yes $=1, \quad \mathrm{No}=0)+0.45 \times \mathrm{T} 2 \mathrm{DM} \quad(\mathrm{Yes}=2, \quad \mathrm{No}=0)+$ $0.15 \times \mathrm{I} 0+0.04 \times \mathrm{AST}-0.94 \times \mathrm{AST} / \mathrm{ALT}$ [I0, fasting insulin $(\mu \mathrm{U} / \mathrm{ml})$; T2DM, type $2 \mathrm{DM}$; AST, ALT representing fasting AST and ALT (U/L), respectively] [15].

Measurement of CIMT was done using a $7.5-\mathrm{MHz}$ linear array transducer where the common carotid, the carotid bulb, and the near and far wall segments of the internal carotid arteries were scanned bilaterally according to the consensus statement from the American Society of Echocardiography. Examination was done in the supine position with the head tilted $45^{\circ}$ contralaterally. CIMT was defined as the distance between the lumen-intima and the media-adventitia ultrasound interfaces. Measurements were consisted of six manual measurements at equal distances along $1 \mathrm{~cm}$ on the far wall of common carotid artery. Left and right CIMT were averaged (Fig. 1).

Abdominal ultrasonography was done after an overnight fasting by two experienced radiologists who were blind to patients' clinical characteristics. It was done using a Toshiba Nemio XG (Toshiba Medical Systems Corporation Europe B. V, Japan) with a 4.5-MHz frequency convex probe and a high-resolution B-mode scanner with patients in supine position, with special emphasis on liver span of the right lobe in the mid-clavicular line on oblique view and liver echogenicity. Increased liver echogenicity rather than the kidney and either attenuation of ultrasound signal of the liver with the diaphragm indistinct or the echogenic walls of the portal veins were less visible were the indicators of NAFLD (Fig. 2). The degree of fatty liver was scored as shown in Table 1. Cases were classified to have mild (1-3), moderate (4-6), and severe $(\geq 7)$ fatty liver changes [16].

Abdominal MRI was performed within the same week of clinical, laboratory, and sonographic assessment by an experienced MR technologist and analyzed, under the 


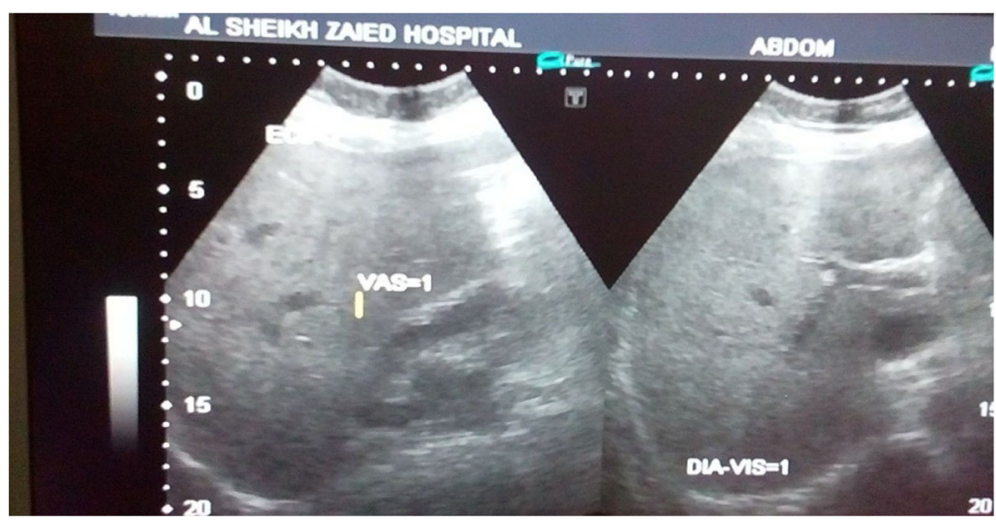

Fig. 1 Doppler ultrasound imaging of the carotid artery showing carotid intima-media thickness measuring $0.8 \mathrm{~mm}$

supervision of the radiology investigator, by a single trained image analyst who was blinded to all data. A 1.5 Tesla MRI (Philips, Achieva) was used for acquisition of liver images. MRI protocols included gradient echo sequences, including in-phase sequence (with TR $10 \mathrm{~ms}$ and TE $4.5 \mathrm{~ms}$ ) and out-phase sequence (with TR $10 \mathrm{~ms}$ and TE $2.5 \mathrm{~ms}$ ) with flip angle $15^{\circ}$. Slice thickness $=5 \mathrm{~mm}$; spacing $=1 \mathrm{~mm}$; acquisition matrix $=256 \times 224$; number of averages $=1$; and acquisition type $=2 \mathrm{D}$.

Echocardiographic assessments were performed by a single experienced examiner, who was blinded to the results of the study group. Tracings were taken with patients in a partial left decubitus position using a VIVID-7 Pro ultrasound machine (GE Technologies, Milwaukee, WI, USA) with an annular phased array $2.5-\mathrm{MHz}$ transducer. The mean values from at least five measurements of each parameter for each patient were computed. Measurements of IVS thickness, PW thickness, and LVID were made at end-

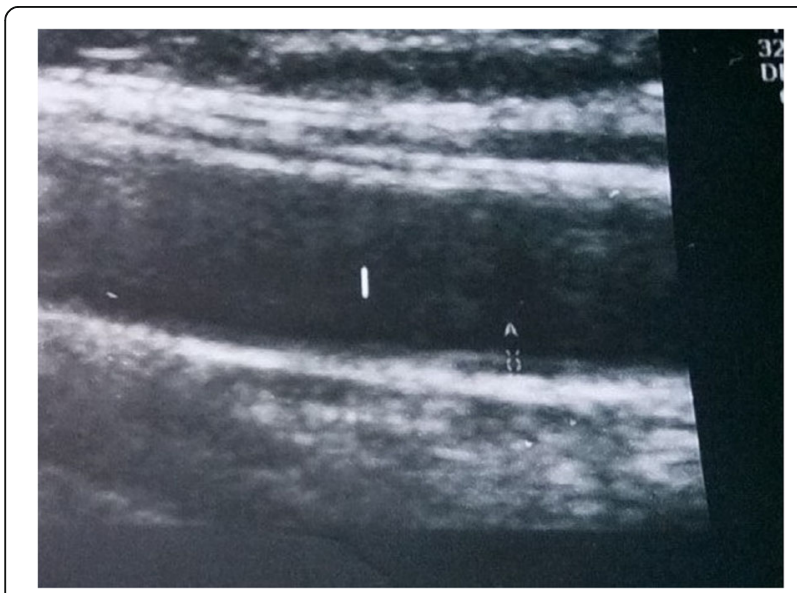

Fig. 2 Imaging of hepatic steatosis scoring by ultrasound showing hepatic echotexture (ECO) scoring 2, clarity of liver blood vessel structures (VAS) scoring 1, and visibility of diaphragm (DIA-VIS) scoring 1; hence, this case is classified to have moderate fatty liver changes (score 4) diastole and end-systole, with special stress up on left ventricular end-diastolic diameter (LVEDD) and left ventricular hypertrophy (LVH).

\section{Statistical analysis}

IBM SPSS statistics (V. 24.0, IBM Corp., USA, 2016) was used for data analysis. Mean \pm SD was used for quantitative parametric data, and median percentiles for quantitative non-parametric data, while number and percentage for categorical variables. Student's $t$ test was used to compare between two independent mean groups for parametric data, and the Mann-Whitney $U$ test was used to compare between two independent groups for non-parametric data. Chi-square test was used to study the comparison between two independent groups as regards the qualitative data.

ROC curve was applied to get the best cutoff values to discriminate patients with NAFLD from healthy controls. Logistic multi-regression analysis was used to search for independent parameters that can predict the target parameter (dependent variable). By using logistic stepwise multi-regression analysis, we can get the most sensitive ones that predict the dependent variable. $p$ value $<0.05$ was considered significant.

\section{Results}

The present study was conducted on 60 subjects with NAFLD including 32 females and 28 males, their ages ranging between 29 and 56 years old, with a mean of $42.1 \pm 6.75$ years, compared to 30 healthy volunteers including 16 males and 14 females, their ages ranging between 28 and 55 years with a mean of $42.06 \pm 8.1$ years.

Clinical and biochemical parameters of all studied subjects are shown in Table 2. Regarding liver profile, hepatic transaminases, serum albumin, and INR showed a significant difference between the 2 groups $(p<0.01$, $p=0.01, p=0.03$, respectively). 
Table 1 Scoring protocol for hepatic steatosis by ultrasonography

\begin{tabular}{|c|c|c|}
\hline U/S features & Score & Definition \\
\hline \multirow[t]{4}{*}{ Liver echotexture (ECO) } & 0 & $\begin{array}{l}\text { Normal: echo level of the liver parenchyma is homogenous } \\
\text { and no difference in contrast between liver and kidney parenchyma }\end{array}$ \\
\hline & 1 & Mild fatty change: slightly increase in echo pattern of the liver \\
\hline & 2 & Moderate fatty change: intermediate between scores 1 and 3 \\
\hline & 3 & $\begin{array}{l}\text { Severe fatty change: gross discrepancy of the increased hepatic } \\
\text { to renal cortical echogenicity }\end{array}$ \\
\hline \multirow[t]{4}{*}{ Echo penetration and visibility of diaphragm (DIA-VIS) } & 0 & $\begin{array}{l}\text { Normal: liver structure is clearly defined from the surface of } \\
\text { the diaphragm. The outline of the diaphragm is clearly visualized }\end{array}$ \\
\hline & 1 & Mild fatty change: mild attenuation of sound beam through the liver \\
\hline & 2 & Moderate fatty change: intermediate between scores 1 and 3 \\
\hline & 3 & $\begin{array}{l}\text { Sever fatty change: marked attenuation of sound beam through } \\
\text { the liver. The diaphragm is not visualized }\end{array}$ \\
\hline \multirow[t]{4}{*}{ Clarity of liver blood vessel structures (VAS) } & 0 & Normal: vessel wall and lumen of the vessel can be clearly visualized \\
\hline & 1 & Mild fatty change: slightly decreased definition of portal venule walls \\
\hline & 2 & Moderate fatty change: intermediate between scores 1 and 3 \\
\hline & 3 & $\begin{array}{l}\text { Sever fatty change: only the main portal veins can be visualized } \\
\text { with the absence of all smaller portal venule walls }\end{array}$ \\
\hline
\end{tabular}

Table 2 Clinical and biochemical parameters of cases and controls

\begin{tabular}{llll}
\hline Variable & Cases $(n=60)$ & $\begin{array}{l}\text { Controls } \\
(n=30)\end{array}$ & $p$ \\
\hline Age (years) & $42.1 \pm 6.75$ & $42.06 \pm 8.1$ & 0.989 \\
SBP (mmHg) & $130.17 \pm 10.3$ & $121.67 \pm 12.2$ & 0.029 \\
DBP (mmHg) & $80.70 \pm 8.6$ & $76.33 \pm 7.7$ & 0.09 \\
ALT (U/L) & $39.2 \pm 9.3$ & $13.5 \pm 3.6$ & $<0.01$ \\
AST (U/L) & $34.1 \pm 6.73$ & $16.07 \pm 3.51$ & $<0.01$ \\
ALP (U/L) & $138.4 \pm 31.7$ & $134.3 \pm 18.0$ & 0.59 \\
T. Bil (mg/dl) & $0.675 \pm 0.4$ & $0.5 \pm 0.4$ & 0.1 \\
D. Bil (mg/dl) & $0.3 \pm 0.2$ & $0.3 \pm 0.35$ & 0.8 \\
Albumin (mg/dl) & $4.05 \pm 0.39$ & $4.43 \pm 0.45$ & 0.01 \\
PT (seconds) & $12.69 \pm 0.82$ & $12.58 \pm 0.42$ & 0.55 \\
INR & $1.06 \pm 0.12$ & $0.99 \pm 0.08$ & 0.03 \\
FBS (mg/dl) & $97.77 \pm 14.17$ & $79.53 \pm 8.28$ & 0.945 \\
HbA1c & $5.35 \pm 1.16$ & $5.09 \pm 0.43$ & 0.282 \\
Fasting insulin ( $\mu$ UU/ml) & $8.75 \pm 5.05$ & $8.6 \pm 1.9$ & 0.866 \\
HOMA-IR & $1.95 \pm 1.8$ & $2 \pm 0.67$ & 0.539 \\
Triglycerides (mg/dl) & $124.37 \pm 29.4$ & $92.8 \pm 21.58$ & $<0.01$ \\
Cholesterol (mg/dl) & $173.3 \pm 40.3$ & $141.3 \pm 15.5$ & $<0.01$ \\
HDL (mg/dl) & $46.2 \pm 7.9$ & $54.7 \pm 7.3$ & 0.001 \\
LDL (mg/dl) & $99.5 \pm 32.5$ & $87 \pm 17$ & $<0.01$ \\
\hline SBP systic blood & &
\end{tabular}

$S B P$ systolic blood pressure, $D B P$ diastolic blood pressure, $A L T$ alanine transaminase, AST aspartate transaminase, T. Bil total bilirubin, D. Bil direct bilirubin, $P T$ prothrombin time, INR international normalized ratio, FBS fasting blood sugar, HbA1c glycated hemoglobin, HOMA-IR homeostasis model assessment-insulin resistance, $H D L$ high-density lipoprotein, $L D L$ low-density lipoprotein
Among cases group, MS and DM were present in 18 cases (30\%) and 14 cases (23.3\%), respectively. Classification of cases using abdominal US to score hepatic steatosis revealed that 30 cases (50\%) had mild steatosis, 24 cases $(40 \%)$ had moderate steatosis, and 6 cases $(10 \%)$ showed severe steatosis. BMI and waist circumference were significantly higher in the NAFLD group $(p<0.01)$ (Table 3). Also, CIMT and NAFLD-LFS and LVEDD were higher in NAFLD patients rather than controls $(p=0.025, p<0.01$, and $p=0.046$, respectively), while $\mathrm{LVH}$ was found in $43(71.67 \%)$ of cases and in 8 $(26.67 \%)$ of controls $(p<0.01)$ (Table 4$)$. On comparing NAFLD patients with and without MS, there was a significant difference as regards TGs $(p=0.028)$, SBP, CIMT, and NAFLD-LFS $(p<0.01)$ (Table 5).

The area under the receiver operating characteristic curve (AUROC) for NAFLD-LFS as a predictor of hepatic steatosis was 0.991 , with a cutoff value of -1.628 as a possible cutoff value to discriminate NAFLD from healthy controls, giving a sensitivity of $96.7 \%$, specificity $100 \%$, PPV100\%, NPV 93.8\%, and diagnostic accuracy of 97.8\% (Fig. 3).

The AUROC for CIMT as a predictor of hepatic steatosis was 0.567 , with a cutoff value of 0.6 as a possible

Table 3 Comparison between cases and controls as regards anthropometric measures

\begin{tabular}{llll}
\hline Anthropometric measures & Cases $(n=60)$ & Controls $(n=30)$ & $p$ \\
\hline BMl $\left(\mathrm{kg} / \mathrm{m}^{2}\right)$ & $34.8 \pm 4.52$ & $25.2 \pm 1.68$ & $<0.01$ \\
WC $(\mathrm{cm})$ & $103.13 \pm 13.62$ & $85.87 \pm 8.97$ & $<0.01$ \\
W/H & $0.96 \pm 0.05$ & $0.91 \pm 0.08$ & 0.09 \\
\hline
\end{tabular}

$B M I$ body mass index, $W C$ waist circumference, $W / H$ waist/hip ratio 
Table 4 Comparison between cases and controls as regards average CIMT, NAFLD-LFS, and echocardiographic parameters

\begin{tabular}{llll}
\hline & $\begin{array}{lll}\text { Cases } \\
(n=60)\end{array}$ & $\begin{array}{l}\text { Controls } \\
(n=30)\end{array}$ & $p$ \\
\hline CIMT (mm) & $0.74 \pm 0.14$ & $0.65 \pm 0.11$ & 0.025 \\
NAFLD-LFS & $-0.94(-1.82$ to 2.57$)$ & $-2.02(-2.57$ to -1.63$)$ & $<0.01^{*}$ \\
LVH & $43(71.67 \%)$ & $8(26.67 \%)$ & $<0.01$ \\
LV EDD (cm) & $5.2 \pm 0.4$ & $4.7 \pm 0.3$ & 0.046 \\
\hline
\end{tabular}

CIMT carotid intima-media thickness, NAFLD-LFS non-alcoholic fatty liver disease-liver fibrosis score, $L V H$ left ventricular hypertrophy, LV EDD left ventricular end-diastolic diameter

*Mann-Whitney (z) test

cutoff value to discriminate NAFLD giving a sensitivity of $70 \%$, specificity $53.3 \%$, PPV $75 \%$, NPV $47.1 \%$, and diagnostic accuracy $64.4 \%$ (Fig. 4). On combining CIMT at 0.7 and NAFLD-LFS at -1.628 as possible cutoff values to discriminate patients with NAFLD from those healthy controls, a sensitivity of $100 \%$, specificity $100 \%$, PPV 100\%, NPV 100\%, and diagnostic accuracy 100\% were reached (Fig. 5).

Finally, logistic stepwise multi-regression analysis revealed that HOMA-IR, total bilirubin, INR, and CIMT were the most sensitive independent predictors of NAFLD (Table 6).

\section{Discussion}

Non-alcoholic fatty liver disease (NAFLD) is reaching epidemic proportions worldwide in parallel with the increasing prevalence of obesity over the past three decades [17]. It has been associated with several risk factors, particularly IR, type II DM, hyperlipidemia, and obesity that are the main features of metabolic syndrome [18].

Routine liver biopsy in such highly prevalent disease seems impractical because of its invasiveness, especially in the presence of imaging techniques with more availability and safety as ultrasound. Unfortunately, ultrasound has limited sensitivity when steatosis is less than $30 \%$ on liver biopsy [7].

Despite its accuracy in quantifying liver fat, especially its advanced techniques like MRI-estimated proton density

Table 5 Comparison between cases with and without MS as regards SBP, serum TGs, and CIMT

\begin{tabular}{llll}
\hline Variable & $\begin{array}{l}\text { Cases with MS } \\
(n=18)\end{array}$ & $\begin{array}{l}\text { Cases without MS } \\
(n=42)\end{array}$ & $p$ \\
\hline TGs $(\mathrm{mg} / \mathrm{dl})$ & $143.9 \pm 29.4$ & $116 \pm 25.8$ & 0.028 \\
SBP $(\mathrm{mmHg})$ & $137.22 \pm 6.18$ & $127.14 \pm 10.316$ & $<0.0$ \\
CIMT $(\mathrm{mm})$ & $0.9 \pm 0.1$ & $0.67 \pm 0.09$ & $<0.01$ \\
NAFLD-LFS & $1.19(-0.5$ to 2.57$)$ & $-1.245(-1.8$ to 1.27$)$ & $<0.01^{*}$
\end{tabular}

MS metabolic syndrome, SBP systolic blood pressure, TGs triglycerides, CIMT carotid intima-media thickness, NAFLD-LFS non-alcoholic fatty liver diseaseliver fibrosis score

*Mann-Whitney (z) test fat fraction (MRI-PDFF) [9], MRI is still time-consuming, relatively expensive, and often unavailable on wide-scale; therefore, there is an urgent need to develop and validate a simple, reproducible, noninvasive method that accurately identifies NAFLD patients with the highest risk of disease progression, meanwhile, allowing frequent monitoring of the disease and response to therapy [10].

Thus, our aim in the current study was to evaluate the clinical value of NAFLD-LFS and CIMT in the prediction of NAFLD in parallel with atherosclerotic cardiovascular disease.

Many serum markers have shown acceptable diagnostic accuracy as defined by an AUROC $>0.8$ [19]. NAFLD fibrosis score (NFS) and fibrosis 4 calculator (FIB-4) have been externally validated in ethnically different NAFLD populations, with consistent results. NFS, FIB-4, enhanced liver fibrosis (ELF), and FibroTest predict overall mortality, cardiovascular mortality, and liver-related mortality. Moreover, NFS can predict incident diabetes, and changes in NFS are associated with mortality. The tests perform best at distinguishing advanced ( $\geq$ F3) versus non-advanced fibrosis but not significant fibrosis $(\geq$ F2) or any fibrosis $(\geq F 1)$ versus no fibrosis. Importantly, the NPV for excluding advanced fibrosis is higher than the corresponding PPV [20]; therefore, noninvasive tests may be confidently used for first-line risk stratification to exclude severe disease. However, predictive values depend on prevalence rates, and most of these studies have been conducted in tertiary centers where the pre-test probability of advanced fibrosis is higher than in the community.

The prevalence and incidence of CVD is higher in NAFLD than in matched controls and linked to the association between NAFLD and MS components [21]. CVD is a main common cause of mortality rather than liver disease itself in NAFLD. In most anecdotal studies, atherosclerotic markers (low HDL, high TG) or inflammatory markers (high sensitive C-reactive protein) and increased levels of procoagulant/prothrombotic factors are more commonly encountered in NAFLD patients than in persons without steatosis. Besides, pre-atherogenic lesions like increased CIMT; coronary artery, abdominal aortic, and aortic valve calcifications; and endothelial dysfunction are also more prevalent in NAFLD patients and, in some studies, correlated with the histological severity [22].

Our study revealed that NAFLD-LFS showed a high diagnostic performance in discriminating patients with NAFLD from healthy controls, with an AUROC for NAFLD-LFS as a predictor of hepatic steatosis of 0.991 . At a value of -1.628 as a possible cutoff value to discriminate NAFLD, it showed a sensitivity of $96.7 \%$, specificity $100 \%$, PPV 100\%, NPV 93.8\%, and diagnostic accuracy $97.8 \%$, a result that is in concordance with Cheung et al. [11] who found that NAFLD-LFS is the best prediction score for ultrasound-proved NAFLD, 


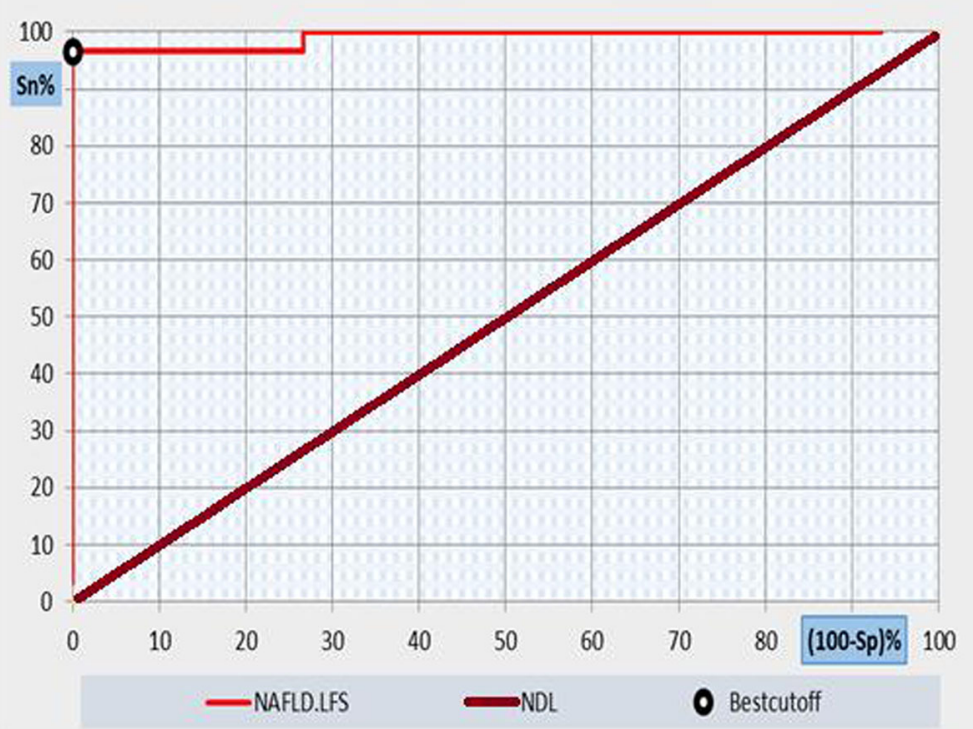

Fig. 3 ROC curve analysis showing the diagnostic performance of NAFLD-LFS in discriminating patients with NAFLD from healthy controls

and can predict mortality, including cardiovascular and liver-related mortality with an AUROC of 0.771 and a high specificity $(96.4 \%)$.

Interestingly, NAFLD-LFS was first developed from a cohort study including 470 well-characterized Finnish individuals, in whom liver fat content was measured using proton magnetic resonance spectroscopy, and it was reported that MS, type II DM, and serum insulin, AST, and ALT concentrations allowed prediction of NAFLD. The score had an AUROC of 0.87 in the estimation and 0.86 in the validation group, and a cutoff point at -0.640 predicted increased liver fat content with an average sensitivity and specificity (86\% and $71 \%$, respectively) [14].

Furthermore, our study revealed higher NAFLD-LFS levels among NAFLD cases than in healthy controls (median -0.94 vs. $-2.02 ; p<0.01$ ). This result was close to that of a retrospective analysis on 324 consecutive liver biopsies performed between 2000 and 2010 for suspicion of NAFLD, where five steatosis biomarkers were calculated including NAFLD-LFS using data retrieved at the time of each liver biopsy, where NAFLD-LFS had higher mean values in grades of mild steatosis than no steatosis, and in moderate than mild steatosis $(p=0.001)$ [23].

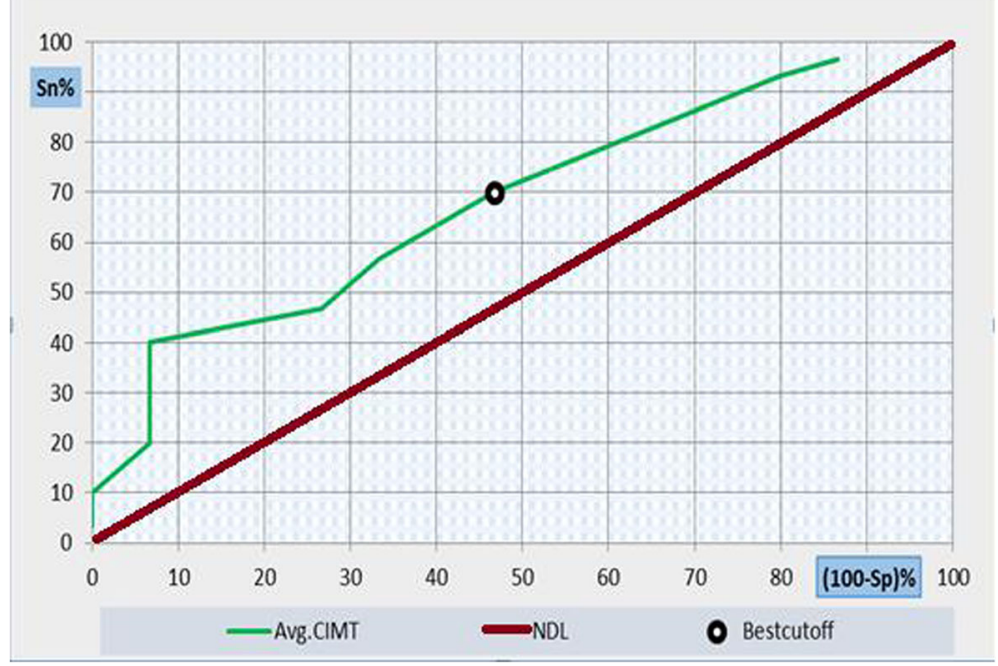

Fig. 4 ROC curve analysis showing the diagnostic performance of CIMT in discriminating patients with NAFLD from healthy controls 


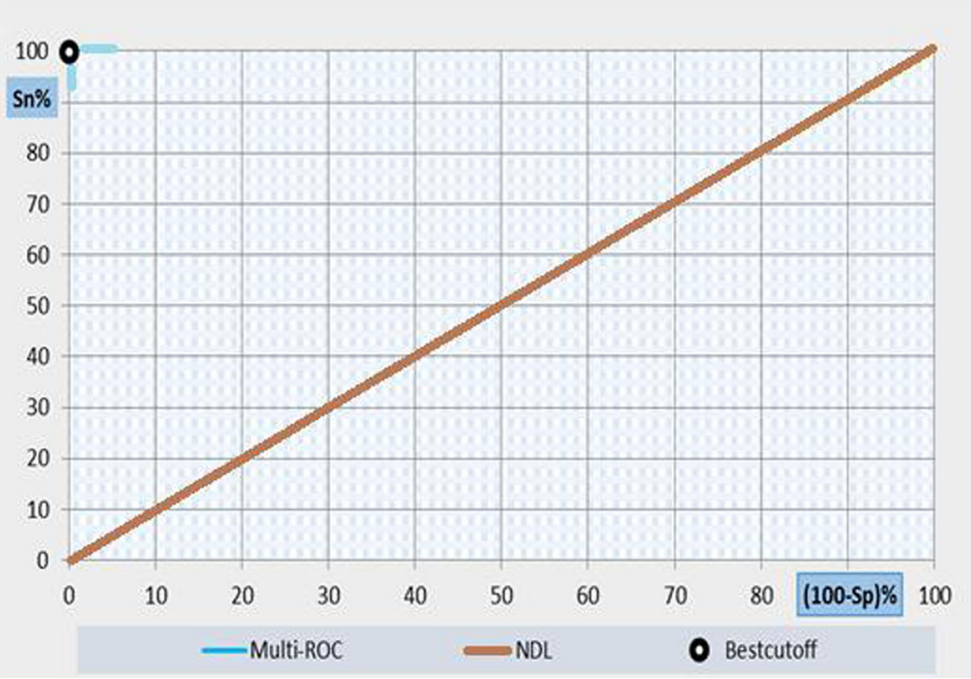

Fig. 5 ROC curve analysis showing the diagnostic performance of combined NAFLD-LFS and CIMT for discriminating patients with NAFLD from healthy controls

Several studies have proven the strong possibility of a carotid plaque in patients with hepatic steatosis [24]; thus, we measured the CIMT in both NAFLD patients and healthy controls to evaluate its clinical value in prediction of NAFLD. Our study revealed that the mean average CIMT among NAFLD cases was significantly higher $(0.74 \pm 0.14 \mathrm{~cm})$ than that of healthy controls $(0.65 \pm 0.11 \mathrm{~cm})(p=0.025)$. Our findings were supported by Fracanzani et al. [25] who evaluated CIMT values in 125 patients with NAFLD and 250 healthy individuals; they found that the mean CIMT was significantly higher in NAFLD patients. Also, our results are comparable with the results of Nahandi et al. [26] who evaluated CIMT values in 49 diabetic NAFLD patients, 50 nondiabetic NAFLD patients, and 52 normal controls, and they reported that there is a significant association between the presence of NAFLD and atherosclerosis determined by CIMT. Gastaldelli and colleagues [27] proved the independent association between FLI and IMT on subjects without diabetes or hypertension in a population-based study including 1307 patients.

Table 6 Multivariate analysis for predictors of NAFLD

\begin{tabular}{llllllll}
\hline Variable & $\begin{array}{l}\text { Reg. } \\
\text { Coeff }\end{array}$ & $p$ & \multicolumn{2}{l}{$95 \% \mathrm{Cl}$} & F-ratio & $p$ \\
\cline { 4 - 5 } & & & Lower bound & Upper bound & & \\
\hline HOMA-IR & 0.76 & $<0.01$ & 0.52 & 1.01 & 19.772 & $<0.001$ \\
T.Bil & 1.97 & 0.02 & 0.39 & 3.55 & & \\
INR & 2.64 & 0.01 & 0.66 & 4.62 & \\
CIMT & 5.2 & 0.11 & -1.183 & 11.589 & \\
\hline
\end{tabular}

Reg. Coeff regression coefficient, HOMA-IR homeostasis model assessmentinsulin resistance, T.Bil total bilirubin, INR international normalized ratio, CIMT carotid intima-media thickness
Also, we found that the mean average CIMT of NAFLD cases with MS was significantly higher $(0.9 \pm 0.1 \mathrm{~cm})$ than that of NAFLD cases without MS $(0.67 \pm 0.9 \mathrm{~cm})(p<0.01)$, and this is in agreement with Koskinen et al. [28], who reported a higher incidence of NAFLD and atherosclerosis among patients with MS. In the contrary, a populationbased study in south Iran, on 290 NAFLD cases compared to 290 healthy controls, suggested that NAFLD could be a risk factor for carotid atherosclerosis, independently of its association with the MS [29].

Furthermore, by multiple regressions analysis, using the stepwise method, HOMA-IR, total bilirubin, INR, and CIMT were together the most sensitive independent variables that predicted the dependent variable NAFLD-LFS.

\section{Conclusion}

NAFLD-LFS might be used as a reliable noninvasive tool for NAFLD diagnosis, while CIMT could not be used solely for NAFLD prediction, but when combined with NAFLD-LFS, they produce a simple and accurate noninvasive tool for diagnosis.

\footnotetext{
Abbreviations

ALP: Alkaline phosphatase; ALT: Alanine aminotransferase; AST: Aspartate aminotransferase; BMI: Body mass index; CIMT: Carotid intima-media thickness; CVD: Cardiovascular disease; DM: Diabetes mellitus; FBG: Fasting blood glucose; Fl: Fasting insulin; FLI: Fatty liver index; HCC: Hepatocellular carcinoma; HDL: High-density lipoprotein; HSI: Hepatic steatosis index; INR: International normalized ratio; LAP: Lipid accumulation product; LDL: Low-density lipoprotein; LFS: Liver fat score; MRI: Magnetic resonance imaging; MS: Metabolic syndrome; NAFLD: Non-alcoholic fatty liver disease; NASH: Non-alcoholic steatohepatitis; PT: Prothrombin time; TC: Total cholesterol; TG: Triglycerides
} 


\section{Acknowledgements}

None.

\section{Authors' contributions}

NM had selected the idea and design of the work and had made the final revision of data, ES had contributed in the data collection and interpretation and shared in drafting the work, SA and AF had helped in the data collection and analysis, and WH had helped in the data acquisition and analysis. All authors have read and approved the final manuscript.

\section{Funding}

This research did not receive any specific grant from funding agencies in the public, commercial, or not-for-profit sectors.

\section{Availability of data and materials}

The datasets generated and/or analyzed during the current study are not publicly available due to confidential and institutional ethical issues but are available from the corresponding author on reasonable request.

\section{Ethics approval and consent to participate}

The study was conducted in accordance with the ethical principles of the 1975 Declaration of Helsinki, and it was reviewed and approved by Research Ethics Committee of Faculty of Medicine, Ain Shams University Institutional Review Board. A written informed consent was obtained from all the participants after explaining the aim and concerns of the study.

\section{Consent for publication}

Not applicable.

\section{Competing interests}

The authors declare that they have no competing interests.

\section{Author details}

${ }^{1}$ Department of Internal Medicine, Faculty of Medicine, Ain Shams University, Khalifa El-Maamon St., Abbassia, Cairo 11341, Egypt. ²Department of Tropical Medicine, Faculty of Medicine, Ain Shams University, Cairo 11341, Egypt. ${ }^{3}$ Department of Radiodiagnosis and Interventional Radiology, Faculty of Medicine, Ain Shams University, Cairo 11341, Egypt.

Received: 21 July 2019 Accepted: 30 July 2019

Published online: 19 September 2019

\section{References}

1. Fargion S, Porzio M, Fracanzani AL (2014) Nonalcoholic fatty liver disease and vascular disease: state-of-the-art. World J Gastroenterol 20(37):13306-13324

2. Vuppalanchi R, Chalasani N (2016) Screening strategies for nonalcoholic steatohepatitis in high-risk individuals: trimming away the fat. Dig Dis Sci 61:1790-1792

3. Zelber-Sagi S, Webb M, Assy N, Blendis L, Yeshua H, Leshno M, Santo E (2013) Comparison of fatty liver index with noninvasive methods for steatosis detection and quantification. World J Gastroenterol 19:57-64

4. Vanni E, Bugianesi E, Kotranen A et al (2010) From the metabolic syndrome to NAFLD or vice versa. Dig Liver Dis 42:320-330

5. Lonardo A, Sookoian S, Chonchol M et al (2013) Cardiovascular and systemic risk in non alcoholic fatty liver disease - atherosclerosis as a major player in the natural course of NAFLD. Curr Pharm Des 19:5177-5192

6. Anstee OM, Targher G, Day CP (2013) Progression of NAFLD to diabetes mellitus, cardiovascular disease or cirrhosis. Nat Rev Gasteroenterol Hepatol 10:330-344

7. Sumida Y, Nakajima A, Itoh Y (2014) Limitations of liver biopsy and noninvasive diagnostic tests for the diagnosis of nonalcoholic fatty liver disease/nonalcoholic steatohepatitis. World J Gastroenterol 20(2):475-485

8. Bohte AE, Van Werven JR, Bipat $S$ et al (2011) The diagnostic accuracy of US, $\mathrm{CT}, \mathrm{MRI}$, and $1 \mathrm{H}-\mathrm{MRS}$ for the evaluation of hepatic steatosis compared with liver biopsy: a meta-analysis. Eur Radiol 21(1):87-97

9. Noureddin M, Lam J, Peterson MR, Middleton M, Hamilton G, Le T et al (2013) Utility of magnetic resonance imaging versus histology for quantifying changes in liver fat in non alcoholic fatty liver disease trials. Hepatology 58(6):1930-1940
10. Bedogni G, Kahn HS, Bellentani S, Tiribelli C (2010) A simple index of lipid overaccumulation is a good marker of liver steatosis. BMC Gastroenterol 10:98

11. Cheung C, Lam KS, Wong IC et al (2014) Non-invasive score identifies ultrasonography diagnosed non alcoholic fatty liver disease and predicts mortality in the USA. BMC Med 12:154

12. American Diabetes Association Diabetes Care 2016; 39(Supplement 1): S13-S22 https://doi.org/10.2337/dc16-S005

13. Muniyappa R, Lee S, Chen H, Quon MJ (2008) Current approaches for assessing insulin sensitivity and resistance in vivo: advantages, limitations, and appropriate usage. Am J Physiol Endocrinol Metab 294:15-26

14. Alberti KG, Eckel RH, Grundy SM, Zimmet PZ, Cleeman Jl, Donato KA et al (2009) Harmonizing the metabolic syndrome: a joint interim statement of the International Diabetes Federation Task Force on Epidemiology and Prevention; National Heart, Lung, and Blood Institute; American Heart Association; World Heart Federation; International Atherosclerosis Society; and International Association for the Study of Obesity. Circulation 120(16):1640-1645

15. Kotronen A, Peltonen M, Hakkarainen A, Sevastianova K, Bergholm R, Johansson LM et al (2009) Prediction of non-alcoholic fatty liver disease and liver fat using metabolic and genetic factors. Gastroenterology 137:865-872

16. Chan D, Li AM, Chu WC, Chan MH, Wong EM, Liu EK et al (2004) Hepatic steatosis in obese children. Int J Obes 28:1257-1263

17. NCD Risk Factor Collaboration (NCD-RisC) (2016) Trends in adult body-mass index in 200 countries from 1975 to 2014: a pooled analysis of 1698 population-based measurement studies with $19 \cdot 2$ million participants. Lancet. 387(10026):1377-1396

18. EASL-EASD-EASO. European Association for the Study of the Liver, European Association for the Study of Diabetes, and European Association for the Study of Obesity (2016) Clinical practice guidelines for the management of non-alcoholic fatty liver disease. J Hepatol 64:1388-1402

19. EASL-ALEH. European Association for the Study of the Liver, Asociacion Latinoamericana para el Estudio del Higado (2015) Clinical practice guidelines: non-invasive tests for evaluation of liver disease severity and prognosis. J Hepatol 63:237-264

20. McPherson S, Hardy T, Henderson E, Burt AD, Day CP, Anstee QM (2015) Evidence of NAFLD progression from steatosis to fibrosing-steatohepatitis using paired biopsies: implications for prognosis and clinical management. J Hepatol 62:1148-1155

21. Oni ET, Agatston AS, Blaha MJ, Fialkow J, Cury R, Sposito A et al (2013) A systematic review: burden and severity of subclinical cardiovascular disease among those with nonalcoholic fatty liver; should we care? Atherosclerosis 230:258-267

22. Targher G, Day CP, Bonora E (2010) Risk of cardiovascular disease in patients with nonalcoholic fatty liver disease. N Engl J Med 363:1341-1350

23. Fedchuk L, Nascimbeni F, Pais R, Charlotte F, Housset C, Ratziu V et al (2014) Performance and limitations of steatosis biomarkers in patients with nonalcoholic fatty liver disease. Aliment Pharmacol Ther 40:1209-1222

24. Mohammadi A, Bazzazi A, Ghamesi-Rad M (2011) Evaluation of atherosclerotic findings in patients with nonalcoholic fatty liver disease. Int $J$ Gen Med 4:717-722

25. Fracanzani AL, Burdick L, Raselli S, Pedotti P, Grigore L, Santorelli G et al (2008) Carotid artery intima-media thickness in nonalcoholic fatty liver disease. Am J Med 121(1):72-78

26. Nahandi MZ, Khoshbaten M, Ramazanzadeh E, Abbaszadeh L, Javadrashid R Shirazi KM et al (2014) Effect of non alcoholic fatty liver disease on carotid artery intima-media thickness as a risk factor for atherosclerosis. Gastroenterol Hepatol Bed Bench 7(1):55-62

27. Gastaldelli A, KozakovaM HK, Flyvbjerg A, Favuzzi A, Mitrakou A et a (2009) Fatty liver is associated with insulin resistance, risk of coronary heart disease, and early atherosclerosis in a large European population. Hepatology 49:1537-1544

28. Koskinen J, Magnussen CG, Kähönen M, Loo BM, Marniemi J et al (2012) Association of liver enzymes with metabolic syndrome and carotid atherosclerosis in young adults. The Cardiovascular Risk in Young Finns Study. Ann Med 44:187-195

29. Lankarani KB, Ghaffarpasand F, Mahmoodi M et al (2013) Non alcoholic fatty liver disease in southern Iran: a population based study. Hepat Mon 13(5):e9248

\section{Publisher's Note}

Springer Nature remains neutral with regard to jurisdictional claims in published maps and institutional affiliations. 